\title{
Stochastic modeling of a Single-Unit Repairable System with Preventive Maintenance under Warranty
}

\author{
Ramniwas \\ Department of Statistics \& O.R., \\ Kurukshetra University, \\ Kurukshetra (India)
}

\author{
M.S. Kadyan \\ Department of Statistics \& O.R., \\ Kurukshetra University, \\ Kurukshetra (India)
}

\author{
Jitender Kumar \\ Department of Statistics \&O.R., \\ Kurukshetra University, \\ Kurukshetra (India)
}

\begin{abstract}
In this paper a stochastic model for a single unit repairable system with Preventive Maintenance (PM) under warranty is analyzed in details by using supplementary variable technique.The cost of repair during warranty is born by the manufacturers, but warranty does not apply to product failure due to user-induced damage such as cracked screen or cracked casing, accident, misuse, physical damage, damage due to liquid and unauthorized modifications etc. within warranty period. Unit goes under PM during warranty and works as new after PM. There is a single server who always remains with the system. The time to failure of the system follows negative exponential distribution while PM and repair time distributions are taken as arbitrary. The expressions for some economic measures such as reliability, mean time to system failure (MTSF) and availability have been derived.A particular case is considered to highlight the behaviour of reliability and profit function.
\end{abstract}

Keywords: Stochastic model, Single-Unit System, Warranty, Preventive Maintenance and Reliability.

\section{INTRODUCTION}

Single-unit systems have been widely studied in the literatue of reliability due to their frequent use in mordern bussiness and industries. Many researchers including Arekar et al. [1], Kadyan et al. [3], Kadyan and Promila [4], Kharoufeh J.P. et al. [5], Malik et al. [6], Proctor and Singh [7], Shakuntla et al. [8] and Uematsu and Nishida [9] have analysed single-unit sysytems under a common assumption that the unit works continuously till failure without undergoing PM.

The continued operation of the systems may reduce performance and reliability of the system.Therefore, PM of the unit is necessary after a specific period of time at any stage of operation to improve the reliability and availability of the system because the cost to repair the system after its failure is greater than the cost of maintaining the system before its failure. Jin et al. [10] have studied reliability models with PM and without considering any warranty and service contract. But, warranty acts as an insurance in the event of an early failure of the product. Better warranty terms serve as an indicator of the reliability of the product and may increase sales.
However, the concept of single-unit system with PM under warranty has not appeared in the literature so far

Thus, in view of the above observations here we analyzed a single unit repairable system with PM under warranty by using supplementary variable technique.The cost of repair during warranty is born by the manufacturers, but warranty does not apply to product failure due to user-induced damage such as cracked screen or cracked casing, accident, misuse, physical damage, damage due to liquid and unauthorized modifications etc. within warranty period. Unit goes under PM during warranty and works as new after PM. There is a single server who always remains with the system. The time to failure of the system follows negative exponential distribution while PM and repair time distributions are taken as arbitrary. The expressions for some economic measures such as reliability, MTSF and availability have been derived. A particular case is considered to highlight the behaviour of reliability and profit function.

\section{MODEL ASSUMPTIONS}

(1) The system has a single unit

(2) There is single server, which is always available with the system.

(3) The repair of the unit within warranty is born by the manufacturer.

(4) Warranty does not apply to product failure due to user-induced damage within warranty period.

(5) PM is made during warranty period.

(6) The unit works as new after repair and PM.

(7) The distribution of failure time is taken as negative exponential while the PM and repair time are considered as arbitrary.

(8) Switching is perfect.

\section{MODEL DEVELOPMENT}

\subsection{State-Specification}

$s_{0} / s_{1}$ The unit is operative under warranty period/ beyond warranty period.

$s_{3} / s_{4}$ The unit is in failed state under warranty period/ beyond warranty period. 
$\boldsymbol{S}_{2}$ The unit is under PM.

\subsection{Notations}

$\lambda / \lambda_{1}$ Constant failure rate of the unit within warranty period/beyond warranty period.

$\alpha \quad$ Constant rate of completion of warranty period.

$\lambda_{m} \quad$ Transition rate which transits the operative unit under PM.

$\mu(x), s(x) / \mu_{1}(x), s_{1}(x) \quad$ Repair rate of the unit and probability density function, for the elapsed repair time ' $x$ ' in warranty period/ beyond warranty period.

$\mu_{2}(y), s_{2}(y)$ PM rate of the unit and probability density function, for the elapsed PM time ' $y$ '.

$p_{0}(t) / p_{1}(t)$ The Probability that at time $t$ the system is in good state in warranty period/ beyond warranty period.

$p_{3}(x, t) \Delta / p_{4}(x, t) \Delta \quad$ The Probability that at time $\mathrm{t}$ the system is in failed state in warranty period/ beyond warranty period, the repair time lies in the interval $(\mathrm{x}, \mathrm{x}+\Delta)$.

$p_{2}(y, t) \Delta$

$p(s)$

$s_{2}(y)$

$s_{1}(x)$ $s(x)$

$\int$

Using the probabilistic arguments and limiting transitions, we have the following difference-differential equations (Cox D.R. [2]):

$$
\begin{aligned}
& {\left[\frac{\partial}{\partial t}+\lambda+\alpha+\lambda_{m}\right] p_{0}(t)=\int \mu(x) p_{3}(x, t) d x+\int \mu_{2}(y) p_{2}(y, t) d y} \\
& {\left[\frac{\partial}{\partial t}+\lambda_{1}\right] p_{1}(t)=\alpha p_{0}(t)+\int \mu_{1}(x) p_{4}(x, t) d x} \\
& {\left[\frac{\partial}{\partial t}+\frac{\partial}{\partial x}+\mu(x)\right] p_{3}(x, t)=0} \\
& {\left[\frac{\partial}{\partial t}+\frac{\partial}{\partial y}+\mu_{2}(y)\right] p_{2}(y, t)=0} \\
& {\left[\frac{\partial}{\partial t}+\frac{\partial}{\partial x}+\mu_{1}(x)\right] p_{4}(x, t)=0}
\end{aligned}
$$

The boundary and initial conditions to be satisfied are given below Boundary conditions

$$
\begin{aligned}
& p_{3}(0, t)=\lambda p_{0}(t) \\
& p_{2}(0, t)=\lambda_{m} p_{0}(t) \\
& p_{4}(0, t)=\lambda_{1} p_{1}(t)
\end{aligned}
$$

Initial conditions

$$
\begin{aligned}
& p_{i}(0)=1 \text {; when } i=0 \\
& p_{i}(0)=0 \text {; when } i \neq 0
\end{aligned}
$$

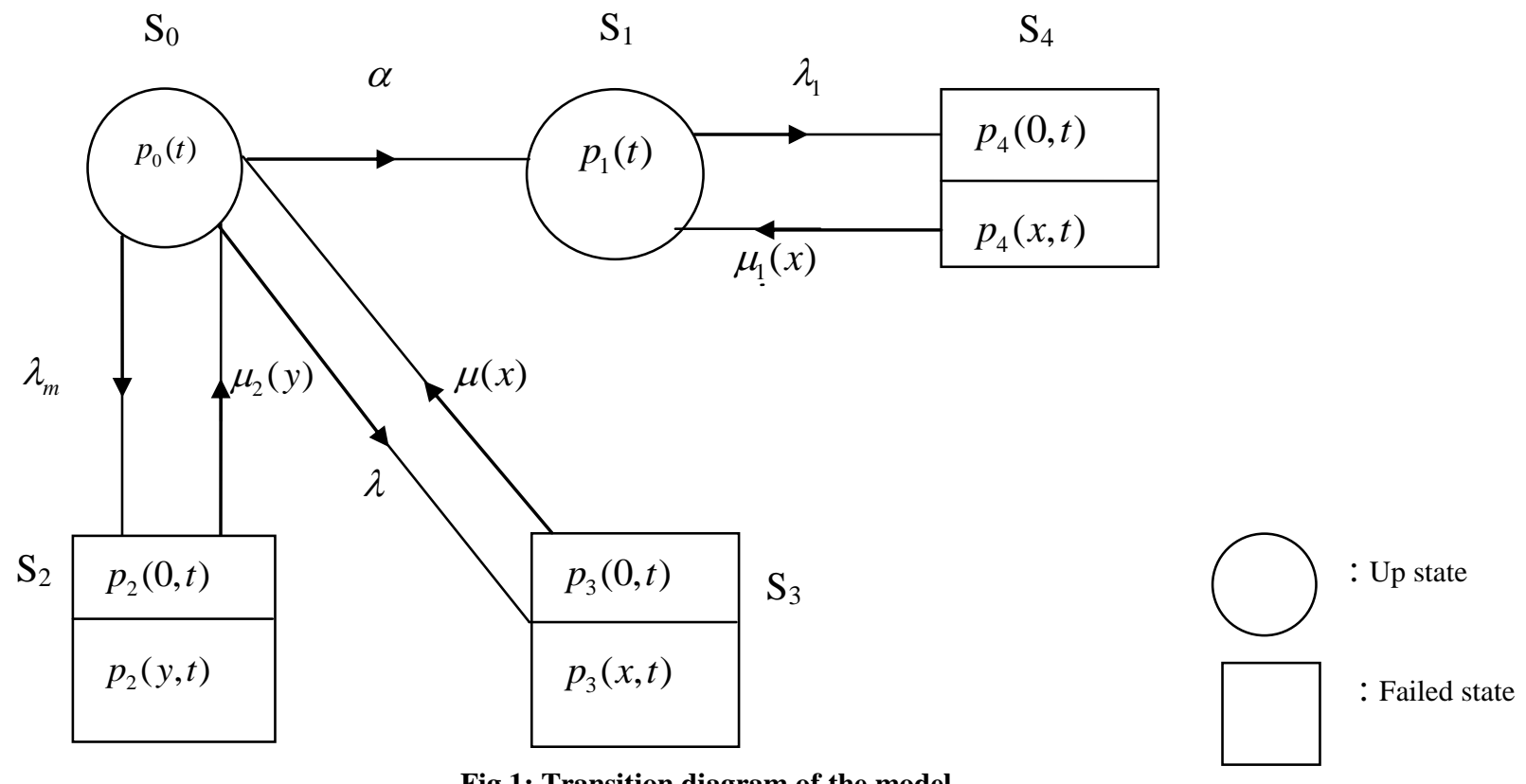

Fig 1: Transition diagram of the model 


\section{MODEL ANALYSIS}

\subsection{Solution of the equations}

Taking Laplace transforms of equations (1)-(8) and using (9) we obtain

$\left[s+\lambda+\alpha+\lambda_{m}\right] p_{0}(s)=1+\int \mu(x) p_{3}(x, s) d x$

$$
\begin{aligned}
& \quad+\int \mu_{2}(y) p_{2}(y, s) d y \\
& {\left[s+\lambda_{1}\right] p_{1}(s)=\alpha p_{0}(s)+\int \mu_{1}(x) p_{4}(x, s) d x} \\
& {\left[\frac{\partial}{\partial x}+s+\mu(x)\right] p_{3}(x, s)=0} \\
& {\left[\frac{\partial}{\partial y}+s+\mu_{2}(y)\right] p_{2}(y, s)=0} \\
& {\left[\frac{\partial}{\partial x}+s+\mu_{1}(x)\right] p_{4}(x, s)=0} \\
& p_{3}(0, s)=\lambda p_{0}(s) \\
& p_{2}(0, s)=\lambda_{m} p_{0}(s) \\
& p_{4}(0, s)=\lambda_{1} p_{1}(s)
\end{aligned}
$$

Integrating equation (12) and further using (15) we get

$$
p_{3}(x, s)=p_{3}(0, s) e^{\left[-s x-\int_{0}^{x} \mu(x) d x\right]}
$$

Similarly integrating equation (13) and further using (16) we get

$$
p_{2}(y, s)=p_{2}(0, s) e^{\left[-s y-\int_{0}^{y} \mu_{2}(y) d y\right]}
$$

Similarly integrating equation (14) and further using (17) we get

$$
p_{4}(x, s)=p_{4}(0, s) e^{\left[-s x-\int_{0}^{x} \mu_{1}(x) d x\right]}
$$

Using equations (16) and (19), equation (10) yields

$$
\begin{aligned}
{[s+\lambda+} & \left.\alpha+\lambda_{m}\right] p_{0}(s)=1+p_{3}(0, s) \int \mu(x) p_{3}(x, s) e^{\left[-s x-\int_{0}^{x} \mu(x) d x\right]} d x \\
& +\int p_{2}(0, s) e^{\left[-s y-\int_{0}^{x} \mu_{2}(y) d y\right]} \mu_{2}(y) d y=1+\lambda p_{0}(s) S(s) \\
& +\lambda_{m} p_{0}(s) S_{2}(s) \\
p_{0}(s)= & \frac{1}{T(s)}
\end{aligned}
$$

where $T(s)=s+\alpha+\lambda(1-S(s))+\lambda_{m}\left(1-S_{2}(s)\right)$

Using equations (16)-(17) and (19)-(20), equation (11) yields

$\left.\left[s+\lambda_{1}\right] p_{1}(s)=\alpha p_{0}(s)+p_{4}(0, s) \int \mu_{1}(x) e^{\left[-s x-\int_{0}^{x} \mu_{1}(x) d x\right.}\right] d x$

$$
=\alpha p_{0}(s)+\lambda_{1} p_{1}(s) S_{1}(s)
$$

$p_{1}(s)=\frac{A(s)}{T(s)}$

where $A(s)=\frac{\alpha}{\left(s+\lambda_{1}-\lambda_{1} S_{1}(s)\right)}$

Now, the Laplace transform of the probability that the system is in the failed state is given by

$$
\begin{aligned}
& p_{3}(s)=\int p_{3}(s, x) d x=\lambda p_{0}(s) \frac{(1-S(s))}{s} \\
& p_{3}(s)=\frac{\lambda B(s)}{T(s)}
\end{aligned}
$$

where $B(s)=\frac{(1-S(s))}{s}$

Similarly $\quad p_{2}(s)=\int p_{2}(s, y) d y=\lambda_{m} p_{1}(s) \frac{\left(1-S_{2}(s)\right)}{s}$

$p_{2}(s)=\frac{\left(\lambda_{m} A(s) C(s)\right)}{T(s)}$

where $C(s)=\frac{\left(1-S_{2}(s)\right)}{s}$

Similarly $p_{4}(s)=\int p_{4}(s, x) d x=\lambda_{1} p_{1}(s) \frac{\left(1-S_{1}(s)\right)}{s}$

$p_{4}(s)=\frac{\left(\lambda_{1} A(s) D(s)\right)}{T(s)}$

where $D(s)=\frac{\left(1-S_{1}(s)\right)}{s}$

It is worth noticing that

$p_{0}(s)+p_{1}(s)+p_{2}(s)+p_{3}(s)+p_{4}(s)=\frac{1}{s}$

\subsection{Evaluation of Laplace transforms of up anddown state probabilities}

The Laplace transforms of the probabilities that the system is in up (i.e. good) and down (i.e. failed) state at time " $t$ " are as follows

$A v(s)$ or $P_{u p}(s)=p_{0}(s)+p_{1}(s)$

$$
\begin{aligned}
& A v(s)=\frac{(1+A(s))}{T(s)} \\
& P_{\text {down }}(s)=p_{2}(s)+p_{3}(s)+p_{4}(s) \\
& P_{\text {down }}(s)=\frac{\left(\lambda B(s)+\lambda_{1} A(s) D(s)+\lambda_{m} A(s) C(s)\right)}{T(s)}
\end{aligned}
$$

\subsection{Steady-State Probabilities}

Using Abel's Lemma in Laplace transforms, viz.

$\lim _{s \rightarrow 0} s[Z(s)]=\lim _{n \rightarrow \infty}[Z(t)]=Z($ say $)$,

Provided the limit on the right hand side exists, the following time independent probabilities have been obtained.

$$
\begin{aligned}
& A v=\frac{1}{\left(1-\lambda_{1} S_{1}^{\prime}(0)\right)} \\
& P_{\text {down }}=\frac{\left(-\lambda_{1} S_{1}^{\prime}(0)\right)}{\left(1-\lambda_{1} S_{1}^{\prime}(0)\right)}
\end{aligned}
$$

\subsection{Reliability Indices}

In order to obtain system reliability, consider repair rates $\left(\right.$ i.e., $\left.\mu(x), \mu_{1}(x)\right)$ and PM rate $\mu_{2}(y)$ equal to zero. Using the method similar to that in section 3 , the differential-difference equations are:

$$
\begin{aligned}
& {\left[\frac{\partial}{\partial t}+\lambda+\alpha+\lambda_{m}\right] p_{0}(t)=0} \\
& {\left[\frac{\partial}{\partial t}+\lambda_{1}\right] p_{1}(t)=\alpha p_{0}(t)}
\end{aligned}
$$


Theorem 1. The reliability of the system is given by

$$
\begin{aligned}
R(t)= & e^{-\left(\lambda+\alpha+\lambda_{m}\right) t}\left[\frac{\left(\lambda-\lambda_{1}+\lambda_{m}\right)}{\left(\lambda-\lambda_{1}+\lambda_{m}+\alpha\right)}\right] \\
& e^{-\left(\lambda_{1}\right) t}\left[\frac{\alpha}{\left(\lambda-\lambda_{1}+\lambda_{m}+\alpha\right)}\right]
\end{aligned}
$$

Proof. Taking Laplace transforms of (38) and (39) and using (9) we get

$\left[s+\lambda+\alpha+\lambda_{m}\right] p_{0}(s)=1$

$\left[s+\lambda_{1}\right] p_{1}(s)=\alpha p_{0}(s)$

Using the initial conditions, the solution can be written as

$$
\begin{aligned}
p_{0}(s) & =\frac{1}{\left(s+\alpha+\lambda+\lambda_{m}\right)} \\
p_{1}(s) & =\frac{\alpha}{\left(s+\alpha+\lambda+\lambda_{m}\right)\left(s+\lambda_{1}\right)} \\
R(s)= & p_{0}(s)+p_{1}(s) \\
& =\frac{1}{\left(s+\alpha+\lambda+\lambda_{m}\right)}+\frac{\alpha}{\left(s+\alpha+\lambda+\lambda_{m}\right)\left(s+\lambda_{1}\right)}
\end{aligned}
$$

Taking inverse Laplace transform, we get

$$
R(t)=e^{-\left(\lambda+\alpha+\lambda_{m}\right) t}\left[\frac{\left(\lambda+\lambda_{m}-\lambda_{1}\right)}{\left(\lambda+\lambda_{m}-\lambda_{1}+\alpha\right)}\right]+\left[\frac{\alpha}{\left(\lambda+\lambda_{m}-\lambda_{1}+\alpha\right)}\right] e^{-\left(\lambda_{1}\right) t}
$$

Corollary 1.The mean time to system failure (MTSF) is:

$$
\begin{aligned}
\text { MTSF } & =\left[\frac{\left(\lambda-\lambda_{1}+\lambda_{m}\right)}{\left(\lambda-\lambda_{1}+\lambda_{m}+\alpha\right)\left(\lambda+\alpha+\lambda_{m}\right)}\right] \\
& +\left[\frac{\alpha}{\left(\lambda-\lambda_{1}+\lambda_{m}+\alpha\right)\left(\lambda_{1}\right)}\right]
\end{aligned}
$$

Proof. Calculating MTSF $=\int_{0}^{\infty} R(t) d t$

$$
\begin{aligned}
& \operatorname{MTSF}=\int_{0}^{\infty}\left\{e^{-\left(\lambda+\alpha+\lambda_{m}\right) t}\left(\frac{\left(\lambda+\lambda_{m}-\lambda_{1}\right)}{\left(\lambda+\lambda_{m}-\lambda_{1}+\alpha\right)}\right)+\left(\frac{\alpha}{\left(\lambda+\lambda_{m}-\lambda_{1}+\alpha\right)}\right) e^{-\left(\lambda_{1}\right) t}\right\} d t \\
& \operatorname{MTSF}=\left[\frac{\left(\lambda-\lambda_{1}+\lambda_{m}\right)}{\left(\lambda-\lambda_{1}+\lambda_{m}+\alpha\right)\left(\lambda+\alpha+\lambda_{m}\right)}\right] \\
& +\left[\frac{\alpha}{\left(\lambda-\lambda_{1}+\lambda_{m}+\alpha\right)\left(\lambda_{1}\right)}\right]
\end{aligned}
$$

\section{THE WARRANTY COST FOR THE MANUFACTURER AND COST FOR THE USER}

Suppose that the useful life of the system is L and the warranty period $[0, \mathrm{~W}$ ) includes the second and third state, in this case we compute the warranty cost for the manufacturer and cost for the user as follows.

(1) The warranty cost for the manufacturer can be represented by

$$
\begin{aligned}
& C_{M}=C_{R} \int_{0}^{W}(\text { failure rate for the third state }) d t \\
& +C_{P M} \int_{0}^{W}(\text { failure rate for the second state }) d t \\
& C_{M}=C_{R} \int_{0}^{W} \lambda d t+C_{P M} \int_{0}^{W} \lambda_{m} d t=W\left[C_{P M} \lambda_{m}+C_{R} \lambda\right]
\end{aligned}
$$

where $C_{M}$ is the cost for the manufacturer, $C_{R}$ is the repair cost and $C_{P M}$ is the PM cost.

(2) Cost for the user can be represented by

$$
\begin{aligned}
C_{B} & =C_{R} \int_{W}^{L}(\text { failure rate for the fourth state }) d t \\
C_{B} & =C_{R} \int_{W}^{L} \lambda_{1} d t=C_{R} \lambda_{1}(L-W)
\end{aligned}
$$

where $C_{B}$ is the cost for the user.

\section{SPECIAL CASES}

\subsection{Availability}

When repair follows exponential time distribution

Setting $S(s)=\frac{\mu}{(s+\mu)}, S_{1}(s)=\frac{\mu_{1}}{\left(s+\mu_{1}\right)}$ and $S_{2}(s)=\frac{\mu_{2}}{\left(s+\mu_{2}\right)}$

where $\mu$ and $\mu_{1}$ are constant repair rates and $\mu_{2}$ is constant PM rate. Putting these values in equations (22)-(26) we get

$$
p_{0}(s)=\frac{1}{I(s)}
$$

where

$$
\begin{aligned}
& I(s)=\frac{\left(s^{3}+s^{2}\left(\lambda+\alpha+\mu+\lambda_{m}+\mu_{2}\right)+s\left(\lambda_{m} \mu+\mu \alpha+\mu_{2} \alpha+\mu \mu_{2}+\alpha \mu_{2}\right)+\alpha \mu \mu_{2}\right)}{(s+\mu)\left(s+\mu_{2}\right)} \\
& p_{1}(s)=\frac{E(s)}{I(s)} \\
& \text { where } E(s)=\left[\frac{\alpha\left(s+\mu_{1}\right)}{s\left(s+\lambda_{1}+\mu_{1}\right)}\right]
\end{aligned}
$$$$
A v(s) \text { or } P_{u p}(s)=p_{0}(s)+p_{1}(s)
$$$$
=\left[\frac{\left(s^{4}+b_{3} s^{3}+b_{2} s^{2}+b_{1} s+b_{0}\right)}{s\left(s+\lambda_{1}+\mu_{1}\right)\left(s^{3}+s^{2} a_{2}+s a_{1}+a_{0}\right)}\right]
$$

Where $b_{3}=\left(\lambda_{1}+\mu+\alpha+\mu_{1}+\mu_{2}\right)$,

$$
\begin{aligned}
& b_{2}=\left(\lambda_{1} \mu+\mu \alpha+\alpha \mu_{1}+\mu_{1} \mu_{2}+\mu \mu_{2}+\mu \mu_{1}+\lambda_{1} \mu_{2}+\mu_{2} \alpha\right), \\
& b_{1}=\left(\mu \alpha \mu_{1}+\alpha \mu_{1} \mu_{2}+\mu \mu_{1} \mu_{2}+\lambda_{1} \mu \mu_{2}+\mu_{2} \alpha \mu\right) \\
& \text { and } b_{0}=\left(\alpha \mu \mu_{1} \mu_{2}\right) \\
& \text { and } a_{2}=\left(\lambda_{m}+\mu+\mu_{2}+\alpha+\lambda\right) \\
& \qquad a_{1}=\left(\lambda_{m} \mu+\mu \alpha+\mu \mu_{2}+\alpha \mu_{2}+\lambda \mu_{2}\right) \text { and } a_{0}=\left(\alpha \mu \mu_{2}\right)
\end{aligned}
$$

Taking inverse Laplace transforms of equations (46) we get 
$A v(t)=\frac{-\alpha \mu \mu_{1} \mu_{2}}{\left(\lambda_{1}+\mu_{1}\right) z_{1} z_{2} z_{3}}$

$+\left\{\frac{\left(\lambda_{1}+\mu_{1}\right)^{4}-b_{3}\left(\lambda_{1}+\mu_{1}\right)^{3}+b_{2}\left(\lambda_{1}+\mu_{1}\right)^{2}-b_{1}\left(\lambda_{1}+\mu_{1}\right)+b_{0}}{\left(\lambda_{1}+\mu_{1}\right)\left(\lambda_{1}+\mu_{1}+z_{1}\right)\left(\lambda_{1}+\mu_{1}+z_{2}\right)\left(\lambda_{1}+\mu_{1}+z_{3}\right)}\right\}$

$\times e^{-\left(\lambda_{1}+\mu_{1}\right) t}$

$+\left\{\frac{\left(z_{1}^{4}+b_{3} z_{1}^{3}+b_{2} z_{1}^{2}+b_{1} z_{1}+b_{0}\right)}{z_{1}\left(\lambda_{1}+\mu_{1}+z_{1}\right)\left(z_{1}-z_{2}\right)\left(z_{1}-z_{3}\right)}\right\} e^{z_{1} t}$

$-\left\{\frac{\left(z_{2}^{4}+b_{3} z_{2}{ }^{3}+b_{2} z_{2}{ }^{2}+b_{1} z_{2}+b_{0}\right)}{z_{2}\left(\lambda_{1}+\mu_{1}+z_{2}\right)\left(z_{1}-z_{2}\right)\left(z_{2}-z_{3}\right)}\right\} e^{z_{2} t}$

$+\left\{\frac{\left(z_{3}^{4}+b_{3} z_{3}^{3}+b_{2} z_{3}^{2}+b_{1} z_{3}+b_{0}\right)}{z_{3}\left(\lambda_{1}+\mu_{1}+z_{3}\right)\left(z_{1}-z_{3}\right)\left(z_{2}-z_{3}\right)}\right\} e^{z_{3} t}$

$z_{1}, z_{2}$ and $z_{3}$ are three roots of the equation $\left(s^{3}+s^{2} a_{2}+s a_{1}+a_{0}\right)$

\subsection{Cost-benefit analysis of the user}

If $\mathrm{K}_{1}$ is revenue cost per unit time and expected profit $\mathrm{H}(\mathrm{t})$ during the interval $(0, \mathrm{t}]$ is given by

$$
\begin{aligned}
& H(t)=K_{1} \int_{0}^{t} A v(t) d t-C_{B} \\
& \int \frac{-\alpha \mu \mu_{1} \mu_{2} t}{\left(\lambda_{1}+\mu_{1}\right) z_{1} z_{2} z_{3}} \\
& +\left\{\frac{\left(\lambda_{1}+\mu_{1}\right)^{4}-b_{3}\left(\lambda_{1}+\mu_{1}\right)^{3}+b_{2}\left(\lambda_{1}+\mu_{1}\right)^{2}-b_{1}\left(\lambda_{1}+\mu_{1}\right)+b_{0}}{\left(\lambda_{1}+\mu_{1}\right)^{2}\left(\lambda_{1}+\mu_{1}+z_{1}\right)\left(\lambda_{1}+\mu_{1}+z_{2}\right)\left(\lambda_{1}+\mu_{1}+z_{3}\right)}\right\} \\
& \times\left(1-e^{-\left(\lambda_{1}+\mu_{1}\right) t}\right) \\
& =K_{1}\left\{+\left\{\frac{\left(z_{1}^{4}+b_{3} z_{1}^{3}+b_{2} z_{1}^{2}+b_{1} z_{1}+b_{0}\right)}{z_{1}^{2}\left(\lambda_{1}+\mu_{1}+z_{1}\right)\left(z_{1}-z_{2}\right)\left(z_{1}-z_{3}\right)}\right\}\left(e^{z_{1} t}-1\right)\right. \\
& -\left\{\frac{\left(z_{2}^{4}+b_{3} z_{2}^{3}+b_{2} z_{2}^{2}+b_{1} z_{2}+b_{0}\right)}{z_{2}^{2}\left(\lambda_{1}+\mu_{1}+z_{2}\right)\left(z_{1}-z_{2}\right)\left(z_{2}-z_{3}\right)}\right\}\left(e^{z_{2} t}-1\right) \\
& +\left\{\frac{\left(z_{3}^{4}+b_{3} z_{3}^{3}+b_{2} z_{3}^{2}+b_{1} z_{3}+b_{0}\right)}{z_{3}^{2}\left(\lambda_{1}+\mu_{1}+z_{3}\right)\left(z_{1}-z_{3}\right)\left(z_{2}-z_{3}\right)}\right\}\left(e^{z_{3} t}-1\right) \\
& -\left[C_{R} \lambda_{1}(L-W)\right]
\end{aligned}
$$

\section{NUMARICAL ANALYSIS}

Table-1: Effect of failure rate $(\lambda)$ on Reliability $(\mathrm{R}(\mathrm{t}))$

\begin{tabular}{|c|l|l|l|l|l|l|}
\hline $\begin{array}{c}\text { Time } \\
\text { in days }\end{array}$ & $\lambda_{1}$ & $\lambda_{m}$ & $\alpha$ & $\begin{array}{c}\mathrm{R}(\mathrm{t}) \\
\text { for } \lambda=0.01\end{array}$ & $\begin{array}{c}\mathrm{R}(\mathrm{t}) \\
\text { for } \lambda=0.02\end{array}$ & $\begin{array}{c}\mathrm{R}(\mathrm{t}) \\
\text { for } \lambda=0.03\end{array}$ \\
\hline 1 & 0.02 & 0.04 & 0.003 & 0.951273 & 0.941822 & 0.932465 \\
\hline 2 & 0.02 & 0.04 & 0.003 & 0.905003 & 0.887139 & 0.869627 \\
\hline 3 & 0.02 & 0.04 & 0.003 & 0.861066 & 0.835738 & 0.811158 \\
\hline 4 & 0.02 & 0.04 & 0.003 & 0.819342 & 0.787422 & 0.75675 \\
\hline 5 & 0.02 & 0.04 & 0.003 & 0.779718 & 0.742002 & 0.70612 \\
\hline 6 & 0.02 & 0.04 & 0.003 & 0.742086 & 0.699302 & 0.659001 \\
\hline 7 & 0.02 & 0.04 & 0.003 & 0.706345 & 0.659158 & 0.615148 \\
\hline 8 & 0.02 & 0.04 & 0.003 & 0.672398 & 0.621414 & 0.574332 \\
\hline
\end{tabular}

Table-2: Effect of failure rate $\left(\lambda_{1}\right)$ on Reliability $(\mathrm{R}(\mathrm{t}))$

\begin{tabular}{|c|c|c|c|l|l|l|}
\hline $\begin{array}{l}\text { Time } \\
\text { in days }\end{array}$ & $\lambda$ & $\lambda_{m}$ & $\alpha$ & $\begin{array}{c}\mathrm{R}(\mathrm{t}) \\
\text { for } \lambda_{1}=0.01\end{array}$ & $\begin{array}{c}\mathrm{R}(\mathrm{t}) \\
\text { for } \lambda_{1}=0.03\end{array}$ & $\begin{array}{c}\mathrm{R}(\mathrm{t}) \\
\text { for } \lambda_{1}=0.05\end{array}$ \\
\hline 1 & 0.01 & 0.04 & 0.003 & 0.951287 & 0.951258 & 0.951229 \\
\hline 2 & 0.01 & 0.04 & 0.003 & 0.90506 & 0.904947 & 0.904837 \\
\hline 3 & 0.01 & 0.04 & 0.003 & 0.86119 & 0.860944 & 0.860708 \\
\hline 4 & 0.01 & 0.04 & 0.003 & 0.819557 & 0.819133 & 0.818731 \\
\hline 5 & 0.01 & 0.04 & 0.003 & 0.780045 & 0.779402 & 0.778801 \\
\hline 6 & 0.01 & 0.04 & 0.003 & 0.742544 & 0.741646 & 0.740818 \\
\hline 7 & 0.01 & 0.04 & 0.003 & 0.706952 & 0.705767 & 0.704688 \\
\hline 8 & 0.01 & 0.04 & 0.003 & 0.67317 & 0.671668 & 0.67032 \\
\hline
\end{tabular}

Table-3: Effect of transition rate $\left(\lambda_{m}\right)$ on Reliability $(\mathrm{R}(\mathrm{t}))$

\begin{tabular}{|r|l|l|l|l|l|l|}
\hline $\begin{array}{l}\text { Time } \\
\text { in days }\end{array}$ & $\lambda$ & $\lambda_{1}$ & $\alpha$ & $\begin{array}{l}\mathrm{R}(\mathrm{t}) \\
\text { for } \lambda_{m}=0.04\end{array}$ & $\begin{array}{l}\mathrm{R}(\mathrm{t}) \\
\text { for } \lambda_{m}=0.05\end{array}$ & $\begin{array}{l}\mathrm{R}(\mathrm{t}) \\
\text { for } \lambda_{m}=0.06\end{array}$ \\
\hline 1 & 0.01 & 0.02 & 0.003 & 0.951273 & 0.941822 & 0.932465 \\
\hline 2 & 0.01 & 0.02 & 0.003 & 0.905003 & 0.887139 & 0.869627 \\
\hline 3 & 0.01 & 0.02 & 0.003 & 0.861066 & 0.835738 & 0.811158 \\
\hline 4 & 0.01 & 0.02 & 0.003 & 0.819342 & 0.787422 & 0.75675 \\
\hline 5 & 0.01 & 0.02 & 0.003 & 0.779718 & 0.742002 & 0.70612 \\
\hline 6 & 0.01 & 0.02 & 0.003 & 0.742086 & 0.699302 & 0.659001 \\
\hline 7 & 0.01 & 0.02 & 0.003 & 0.706345 & 0.659158 & 0.615148 \\
\hline 8 & 0.01 & 0.02 & 0.003 & 0.672398 & 0.621414 & 0.574332 \\
\hline
\end{tabular}

Table-4: Effect of rate $(\alpha)$ of completion of warranty period on Reliability $(\mathrm{R}(\mathrm{t}))$

\begin{tabular}{|c|c|c|c|c|c|c|}
\hline $\begin{array}{c}\text { Time } \\
\text { in days }\end{array}$ & $\lambda$ & $\lambda_{1}$ & $\lambda_{m}$ & $\begin{array}{c}\mathrm{R}(\mathrm{t}) \\
\text { for } \alpha=0.007\end{array}$ & $\begin{array}{c}\mathrm{R}(\mathrm{t}) \\
\text { for } \alpha=0.005\end{array}$ & $\begin{array}{c}\mathrm{R}(\mathrm{t}) \\
\text { for } \alpha=0.003\end{array}$ \\
\hline 1 & 0.01 & 0.02 & 0.04 & 0.95133 & 0.951301 & 0.951273 \\
\hline 2 & 0.01 & 0.02 & 0.04 & 0.905223 & 0.905113 & 0.905003 \\
\hline 3 & 0.01 & 0.02 & 0.04 & 0.861541 & 0.861304 & 0.861066 \\
\hline 4 & 0.01 & 0.02 & 0.04 & 0.82015 & 0.819747 & 0.819342 \\
\hline 5 & 0.01 & 0.02 & 0.04 & 0.780927 & 0.780324 & 0.779718 \\
\hline 6 & 0.01 & 0.02 & 0.04 & 0.743754 & 0.742923 & 0.742086 \\
\hline 7 & 0.01 & 0.02 & 0.04 & 0.70852 & 0.707437 & 0.706345 \\
\hline 8 & 0.01 & 0.02 & 0.04 & 0.67512 & 0.673766 & 0.672398 \\
\hline
\end{tabular}

Table-5: Effect of repair cost $\left(\mathrm{C}_{\mathrm{R}}\right)$ on Profit of the user $(\mathrm{H}(\mathrm{t}))$

\begin{tabular}{|r|l|l|l|l|l|l|}
\hline \multirow{2}{*}{$\begin{array}{c}\text { Time } \\
\text { in days }\end{array}$} & \multicolumn{6}{|c|}{$\lambda=0.01, \lambda_{1}=0.02, \lambda_{\mathrm{m}}=0.04, \alpha=0.003, \mu=0.1, \mu_{1}=0.1, \mu_{2}=0.3$} \\
\cline { 2 - 7 } & $\mathrm{K}_{1}$ & $\mathrm{~L}$ & $\mathrm{~W}$ & $\begin{array}{c}\mathrm{H}(\mathrm{t}) \\
\text { For } \mathrm{C}_{\mathrm{R}}=150\end{array}$ & $\begin{array}{c}\mathrm{H}(\mathrm{t}) \\
\text { For }_{\mathrm{R}}=100\end{array}$ & $\begin{array}{c}\mathrm{H}(\mathrm{t}) \\
\text { For }_{\mathrm{R}}=50\end{array}$ \\
\hline 1 & 500 & 10 & 3 & 467.7027 & 474.7027 & 481.7027 \\
\hline 2 & 500 & 10 & 3 & 937.9105 & 944.9105 & 951.9105 \\
\hline 3 & 500 & 10 & 3 & 1394.481 & 1401.481 & 1408.481 \\
\hline 4 & 500 & 10 & 3 & 1840.975 & 1847.975 & 1854.975 \\
\hline 5 & 500 & 10 & 3 & 2280.044 & 2287.044 & 2294.044 \\
\hline 6 & 500 & 10 & 3 & 2713.703 & 2720.703 & 2727.703 \\
\hline 7 & 500 & 10 & 3 & 3143.52 & 3150.52 & 3157.52 \\
\hline 8 & 500 & 10 & 3 & 3570.747 & 3577.747 & 3584.747 \\
\hline
\end{tabular}




\section{INTERPRETATION OF THE RESULTS}

Tables 1, 2, 3 and 4 show the behavior of system reliability. Tables 1,2 and 3 indicate that the reliability of the system decreases with the increase of failure rates $(\lambda),\left(\lambda_{1}\right)$ and transition rate $\left(\lambda_{\mathrm{m}}\right)$ with respect to (w.r.t.) time and for fixed values of other parameters. From table 4 it is analyzed that the reliability of the system increases with the decrease of rate of completion of warranty $(\alpha)$ w.r.t. time. Table-5 shows that expected profit $\mathrm{H}(\mathrm{t})$ during the interval $(0, \mathrm{t}]$ increase with the decrease of repair cost $\left(C_{R}\right)$ from 150 to 50 .

\section{CONCLUSION}

From tables 1, 2, 3 and 4, it is concluded that a single unit system with PM under warranty can be made more reliable and profitable to use by the following ways:

1) By decreasing the rate of completion of warranty.

2) By decreasing the repair cost.

Also, PM during the warranty may provide the consumer better product service in the postwarranty period and reduce the cost of repairing the deteriorated product.

\section{REFERENCES}

[1] Arekar Kirti, Ailawadi Satish and Jain Rinku 2012. Reliability modelling for wear out failure period of a single unit system, Journal of Statistical and Econometric Methods, 1(1), 33-41.

[2] Cox D.R. 1962. Renewal theory, John Wiley \& Sons INC, New York.
[3] Kadyan M S, Promila, Jitender Kumar 2013. Reliability modeling of a single-unit system with arbitrary distribution subject to different weather condition. Int J Syst Assur Eng Manag, DOI 10.1007/s13198-013-0168-3.

[4] Kadyan M S, Promila 2012. Reliability and Cost-Benefit Analysis of a Single Unit System with Degradation and Inspection at Different Stages of Failure subject to Weather Conditions. International Journal of Computer Applications, 55(6), 0975 8887.

[5] Kharoufeh J.P., Solo C.J. and Ulukus M.Y. 2010. Semi-Markov models for degradation-based reliability, IIE Transactions, 42, 599-612.

[6] Malik S.C., Chand P. and Singh J. 2008. Stochastic analysis of anoperating system with two types of inspection subject to degradation, journal of applied probability \& statistics, 3(2), 227241.

[7] Proctor C.L., Singh B. 1976. A repairable 3- state device, IEEE Trans. Reliab., R-25, 210-211.

[8] Shakuntla S., A.K. Lal, S.S. Bhatia and Jai Singh 2011. Reliability analysis of polytube industry using supplementary variable technique, Applied Mathematics and Computation, 218, 3981-3992.

[9] Uematsu K and Nishida T 1987. One-unit system with a failure rate depending upon the degree of repair, Mathematica Japonica, 32/1, 139-147

[10] Xiaoning Jin, Lin Li and Jun Ni 2009. Option model for joint production and preventive maintenance system, Int. J. Production Economics, 119, 347-353. 